\title{
Nord Perdu : témoignage de la surconscience linguistique et de l'étrangéité chez Nancy Huston
}

\author{
Juan Carlos AlONSO JuÁREZ \\ Universidad Nacional de Educación a Distancia \\ jalonso417@alumno.uned.es \\ http://orcid.org/0000-0003-3045-7696
}

\section{Resumen}

Este estudio presenta una crítica literaria sobre la hiperconciencia lingüística y cultural -característica a menudo asociada a los escritores migrantes- en Nancy Huston, a través del análisis de sus obras. A partir de un conjunto representativo de Estas, analizamos, con un método integrador, los ensayos de la escritora para buscar en Nord perdu esencialmente (uno de sus ensayos más autobiográficos) las huellas de dicha hiperconciencia, a menudo acompañada de plurilingüismo literario. Los resultados del estudio muestran la existencia de hiperconciencia lingüística y cultural (extrañamiento) en Huston, y especialmente en el ensayo analizado.

Palabras clave: literatura francófona, escritores migrantes, plurilingüismo literario, adquisición de lenguas extranjeras, interculturalidad.

\section{Résumé}

Cet article présente une critique littéraire sur la surconscience linguistique et culturelle - caractéristique souvent associée aux écrivains migrants - chez Nancy Huston, à travers l'analyse de ses œuvres. À partir d'un corpus d'ouvrages représentatifs, nous avons analysé, avec une méthode intégratrice, les essais de l'écrivaine afin d'y rechercher dans Nord perdu essentiellement (l'un de ses essais les plus autobiographiques) les traces de cette surconscience, souvent accompagnée de plurilinguisme littéraire. Les résultats de l'étude prônent l'existence de surconscience linguistique et culturelle (étrangéité) chez Huston, et notamment dans l'essai analysé.

Mots clé : littérature francophone, écrivains migrants, plurilinguisme littéraire, acquisition des langues étrangères, interculturalité.

\begin{abstract}
This article presents a literary criticism of linguistic and cultural hyperawareness -characteristic often associated with migrant writers- in Nancy Huston, through the analysis of her literature. From a corpus of representative works, we analyzed, with an integrative method, the writer's essays in order to search in Nord perdu specially (one of her more
\end{abstract}

\footnotetext{
"Artículo recibido el 19/06/2020, aceptado el 1/03/2021.
} 
autobiographical essays) the traces of the above-mentioned hyperawareness, often accompanied by literary multilingualism. The results of the study pointed out the existence of linguistic and cultural (strangeness) hyperawareness in Huston, and especially in the essay analyzed.

Keywords: francophone literature, migrant writers, literary multilingualism, foreign language acquisition, interculturality.

\section{Introduction}

Serait-il encore artificiel de s'obstiner à séparer littérature francophone et littérature française?

Quoiqu'il en soit, il paraît exister de réelles différences parmi ces écrivains d'écriture française. Actuellement, un des aspects les plus pertinents pour le considérer ainsi, concerne la "production en situation de contacts de langue et de culture : le texte francophone se caractériserait par des indices de ce multilinguisme multiculturel " (Liébois, 2018 : 328) qui résultent généralement de ce que Lise Gauvin (1997 : 6-15) avait déjà appelé " surconscience linguistique » et Nancy Huston "étrangéité " (versant culturel de cette surconscience) (Huston et Sebbar, 1986 : 254).

Nancy Huston (2004: 22) nous confie qu' « il y a nettement plus d'autobiographie dans $[\mathrm{s}]$ es essais que dans [s] es romans ! ». Par conséquent, nous avons consulté, parmi les premiers, des recueils d'articles sur des sujets divers, préalablement parus principalement en revues et, rassemblées ultérieurement en volume, Désirs et réalités (1995), Ames et corps (2004), et surtout Nord perdu (1999) en raison de sa thématique, qui feront partie du corpus d'œuvres analysées à la recherche de la surconscience linguistique et de l'étrangéité chez Huston.

\section{La carrière littéraire de Nancy Huston}

Dans la littérature de Huston (1995 : 264), écrivaine « canadienne et française mais non pas canadienne-française [...], la multiplicité des identités et des voix est une constante " (Armel, 2018 : 165). En 1973, à l'âge de vingt ans, elle émigre à Paris où, bien qu'anglophone, elle réalisera sa prolifique carrière d'écrivaine en langue française principalement. En effet, comme le remarque Anne-Rosine Delbart, " Huston apparaît [...] comme une écrivaine française quoi qu'elle fasse et, même s'il lui arrive d'écrire en anglais, l'original semblera toujours le texte français " (Dion, Lüsebrink et Riesz, 2002 : 49). Et, comme la même Nancy Huston (1995 : 218) nous révèle : « [le français] me "maternait" mieux que ne l'avait jamais fait ma langue maternelle [l'anglais]». Précisons que l'abandon de sa mère marqua profondément Huston : "le lien que j'avais, petite, avec ma mère était un lien d'absence » (Argand, $2001: 35$ ).

Cependant, la première langue étrangère de la petite Nancy ne fut pas le français sinon l'allemand. En effet, à l'âge de six ans, lors du divorce de ses parents, Huston fut abandonnée par sa Mommy (Huston, 1995: 265), puis émigra en 
Allemagne avec son père chez sa mère adoptive, sa Mutti (Huston,1995 : 265). Elle évoquera dans Nord perdu (Huston, 1999a : 74-76) des épisodes cocasses à ce sujet. Ce ne sera qu'en retournant ensuite aux États-Unis pour y faire des études universitaires dans sa langue maternelle, l'anglais, qu'elle apprendra le français pour finalement aller faire des études post-universitaires à Paris, ville qui sera pour elle un « exil joyeusement choisi » (Huston, 1995 : 201).

Dans la ville-lumière, avec le français, lui viendra enfin le besoin d'écrire. Elle en témoigne dans Lettres parisiennes à Leïla Sebbar : « la concomitance temporelle entre l'acquisition du français et la "venue à l'écriture", la première démarche conditionnant même la seconde" (apud Dion, Lüsebrink et Riesz, 2002 : 44). Comme le précise Loraine Day (2007: 8) " a second language may be used as a defence against past trauma and unpalatable present realities " et Christine Klein-Lataud (1996: 214) ajoute dans ce sens, " adopter une langue autre, c'est donc échapper aux pesanteurs de son passé linguistique ». Nancy Huston le déclare sans détour "langue étrangère, nouvelle identité ; l'autre, l'ancienne est jetée à la poubelle " (Huston, 1995 : 265). Et Christine Klein-Lataud (1996:215) à nouveau, nous ôte tout doute à ce propos : «la langue étrangère est doublement libératrice, puisqu'elle libère à la fois de la langue maternelle et du passé avec lequel celle-ci fait bloc ».

Le terrain était donc préparé pour une écriture qui s'avérera féconde. Comme le remarque Bessy (2008 : 91) à propos des écrivains bilingues, "la distanciation linguistique offerte par la langue étrangère ouvre un horizon de créativité inexistant dans la seule langue maternelle ", et " en prenant leurs distances par rapport à ce qui définit leur être profond (langue, milieu, culture, mère, etc.), en s'installant dans l'altérité par la langue, ces auteurs veulent faire émerger un nouveau moi, une subjectivité réinventée " (Bessy, 2008 : 92). Ainsi, en se donnant une langue choisie et non subie, comme dirait Jean-Pierre Castellani (2001), Nancy Huston s'est libérée de manière profonde de son passé, l'écriture en français langue étrangère l'aurait aidé efficacement dans cette entreprise. Ces précisions faites sur le choix du français comme langue d'écriture nous introduisent directement dans ce qui constitue l'objectif de notre travail : la recherche des empreintes de la surconscience linguistique et culturelle chez Nancy Huston.

\section{Cadre conceptuel : la surconscience linguistique et l'étrangéité}

Christiane Albert (1999: 6) remarque que « la spécificité de la littérature francophone tenait en grande partie à la nécessité pour chaque écrivain de définir sa propre langue d'écriture dans un contexte plurilingue ", ce que Lise Gauvin avait déjà appelé "la surconscience linguistique de l'écrivain" (apud Albert, 1999: 6). Selon Gauvin (2003 : 19), " cette surconscience est aussi une conscience de la langue comme espace de fiction voire de friction : soit un imaginaire de et par la langue ». Ainsi, Huston (2004 : 61) le déclare également : «si on est à l'aise, on n'écrit pas : un minimum de 
friction [...] est indispensable à la mise en marche de la machine littéraire ». Lise Gauvin avait déjà mentionné à ce propos que la surconscience linguistique de l'écrivain est une surconscience pour qui « la langue est synonyme d'inconfort et de doute, pour qui la langue est à la fois tourment, laboratoire et exploration " (apud Dion, Lüsebrink et Riesz, 2002 : 24-25).

En outre, nous expliquerons brièvement l'autre concept central présent dans ce travail, celui d'étrangéité. Il s'agit à vrai dire d'un néologisme créé par Nancy Huston pour faire référence à ce sentiment de se sentir étranger dans un autre pays que le sien. Il semblerait que Nancy Huston, à l'âge de 37 ans, " (ait) pris conscience de sa condition d'exilée » (Armel, 2018 : 165). Ainsi, Névine El Nossery (2007 : 393) nous rappelle que « nombreux sont les romans et les essais de Nancy Huston abordant des questions qui la touchent de près, telles l'exil, l'expatriation, le bilinguisme, le sentiment d'étrangeté vécu dans son pays d'accueil et lors de ses brefs retours au pays d'origine ". C'est dans ce contexte que s'inscrit sa quête identitaire ou "d'intensité " (Huston, 1995 : 200). De plus, comme le remarque Névine El Nossery (2007 : 390), " la quête des origines, la problématique de l'exil et de la double appartenance constituent un leitmotiv récurant aussi bien dans ses romans [...] que dans ses essais [...] ». D’ailleurs, pour d'autres écrivains migrants comme Vasillis Alexakis, ce sentiment d'étrangéité devient même un atout : " la nouvelle patrie et la nouvelle langue sont à l'origine de nouveaux thèmes et de nouveaux espaces. La problématique de l'Autre, de l'Étranger, de l'Immigré, de l'Exilé, se répercutent sur l'écriture de l'auteur qui s'enrichit de nouveaux thèmes" (Oktapoda, $2017: 130)$.

\section{La carrière littéraire de Nancy Huston}

Après avoir présenté succinctement le célèbre essai de Huston (Nord perdu), nous examinerons, dans la première partie de ce travail, les implications littéraires de la surconscience linguistique dans les essais de l'écrivaine versant sur la situation d'inconfort ou d'insécurité provoquée par l'utilisation de la langue française, dans les métadiscours et la thématisation sur la langue, ainsi que dans l'utilisation de divers procédés tels que le procès de traduction, l'utilisation d'une autre langue étrangère, voire l'intégration de xénismes.

\subsection{Présentation sommaire de Nord perdu}

Laissons l'écrivaine nous dévoiler l'enjeu de son essai autobiographique Nord perdu (Huston, 1999a : 19) dans la description générale de ce livre :

Ici je vais dire pour la première fois une chose quej'aurai maintes occasions de répéter par la suite, au point qu'on pourrait presque la décrire comme le leitmotiv, l'idée motrice, voire (Dieu m'en garde) le message de ce petit livre, ajoutons-y donc carrément des italiques : l'expatrié découvre de façon consciente (et parfois douloureuse) un certain nombre de réalités qui façonnent, le plus souvent à notre insu, la condition humaine. 
Sans doute veut-elle nous faire part de son expérience. De plus, ce qui intéresse Huston, c'est le culturel et non le naturel. Ainsi, elle écrivit à Leïla Sebbar (1986 : 252) : "vivre à l'étranger m'a permis d'avoir, vis-à-vis du pays d'origine et du pays d'adoption, un petit recul critique : je les perçois l'un et l'autre comme des cultures". Par conséquent, en abolissant le faux naturel de la langue maternelle, les objectifs de Huston sembleraient être ceux de vouloir faire réfléchir le lecteur et de l'aider à considérer que ce qu'il croit être une évidence comme sa propre langue et sa propre culture n'en est qu'une parmi d'autres. Elle encourage donc ses lecteurs à accepter de bon gré la diversité des langues et à réfléchir sur l'importance du respect des migrants.

À présent, analysons avec un peu plus de détail l'essai dans lequel nous étudierons la surconscience linguistique et l'étrangéité chez Huston. Nord perdu est un livre constitué d'enchaînements de chapitres sans entrelacements, avec un intitulé à fonction cataphorique et donnant quelques pistes sur leur contenu. Examinons, tout d'abord, le titre constitué d'un groupe nominal, "Nord perdu", serait-il une adaptation du nom d'un recueil de courtes histoires patriotiques du canadien Bruce Russel True North (1990) que Nancy Huston cite dans Désirs et réalités? En fait, ce Nord perdu par Nancy Huston, c'est le Canada. De plus, se trouve dans Nord perdu une allusion au jeu de mots perdre le Nord, c'est-à-dire, perdre la raison, s'aliéner. En effet, Huston (1999a : 12) précise que perdre le Nord, c'est « oublier ce que l'on avait l'intention de dire, ne plus savoir où l'on est, perdre la tête" et son équivalent anglais to be all abroad (littéralement, être complètement à l'étranger), c'est être "éparpillé de tous côtés ou aux quatre vents mais aussi ; se tromper tout à fait, dérailler complètement, ne plus y être du tout" (Huston, 1999a : 12). Huston fait déjà allusion ici à l'origine de son estitude que nous verrons plus loin :

S’il y a une négritude dans la littérature, il y a certainement une estitude en tant que concept représentant les traits essentiels de l'écrivain exilé, de celui qui arrive de l'Est. Voici les traits essentiels de l'écrivain exilé : l'hésitation et l'ambiguïté ; la recherche de l'espace de l'entre-deux-langues et de l'entre-deux-cultures [...] (Farkas, $2005: 36$ ).

Ces derniers traits sont parfaitement applicables à Huston, exilée de l'Ouest. L'essayiste nous révèle qu'avoir perdu " le Nord, le Grand Nord, le nord vrai, fort et libre " (Huston, 1999a : 15) lui a permis de comprendre que "l'expatrié découvre de façon consciente (et parfois douloureuse) un certain nombre de réalités qui façonnent, le plus souvent à notre insu, la condition humaine " (Huston, 1999a : 19) et que "les exilés sont riches de leurs identités accumulées et contradictoires" (Huston, 1999a: 18). L'estime de cette richesse culturelle mènera Huston à préférer se considérer de "nulle part» (Huston, 1999a: 18). L'estime de cette richesse culturelle mènera Huston à préférer se considérer de «nulle part ( Huston, 1995 : 225). 
Elle dédicace son livre Nord perdu (qu'elle finira d'écrire en 1998) à son frère avec qui elle jouait étant enfant et qui « vit aujourd'hui au Québec, enseigne, écrit et fait des recherches exclusivement en langue française " (Huston, 1995 : 266), et elle y ajoute dans cette dédicace, une citation en anglais de Thomas Stearns Eliot - qui est comme elle, un auteur bilingue (anglais-français) - tirée de East Coker (1940), dévoilant déjà sa préoccupation pour les questions d'identité et d'appartenance, soumises aux aléas des circonstances personnelles, biographiques et généalogiques. Dans East Coker, « el tiempo no sólo es personal sino también biográfico y [...] genealógico : soy porque en mí confluyen sangre, tierra, lengua. Soy consecuencia en una secuencia cuyo origen está más allá de mí » (Folguerá, 1993 : 260).

Comme épigraphe du livre Nord perdu, conçue comme une citation qui manifeste cette attente d'une lecture donnant sens à l'intertexte, on y trouve un poème de Gerard Manley Hopkins, exprimant un remède pour l'âme tourmentée et un autre de Marica Bodrozic faisant allusion à sa qualité d'étrangère (Huston, 1999a : 9). Gerard Manley Hopkins (1844-1889) est d'après la critique l'un des plus originaux poètes anglais. Le poème que Huston reprend d'une manière partielle dans Nord perdu s'intitule My own heart let me more have pity on. Il fait partie des écrits que Hopkins nous laissera après s'être rétabli d'une forte dépression (qui ne lui empêchera pourtant pas de continuer sa création artistique avec Sonnets of Desolation, 1885). Quelle est la raison pour laquelle Huston a choisi de citer ce texte ? C'est probablement l'espoir que Huston a voulu communiquer à ses lecteurs ( À quelque chose malheur est bon »). En ce qui concerne Marica Bodrožić, c'est une écrivaine d'origine croate qui, à dix ans, quitta son pays natal pour rejoindre ses parents en Allemagne. Dans ses écrits, elle évoque le traumatisme lié à l'étrangéité et à la perte de sa langue maternelle. Cependant, comme dans le poème My own heart... de Gerard Manley Hopkins qui nous parle de la lumière au bout du tunnel (Between pie mountains - lights a lovely mile), Marica Bodrožić nous dévoile comment son conflit intérieur se résoudra grâce à la découverte et l'acquisition de la nouvelle langue étrangère. Huston fait également allusion dès le début de son livre avec la citation «JE NE ME PLAIS PAS. Oui » (Huston, 1999a : 11) à Sviatoslav Richter de même qu'elle le fait en parlant de Lara dans son roman Prodige (1999) à " l'écart irréductible entre ce qu'elle est et ce qu'elle aurait voulu être ou ce qu'on attendait qu'elle fût " (apud Dion, Lüsebrink et Riesz, 2002 : 53).

En outre, Huston commence le premier chapitre de son essai en y incluant une citation d'un écrivain français d'origine russe (Huston, 1999a : 11). Comme le souligne Mary Gallagher, Huston «se met à la place de Romain Gary, qui lui aussi répondit à l'abandon maternel par un dédoublement identitaire " (Dvorak et Koustas, 2004 : 31). En tant qu'écrivaine, Huston se sent divisée, comme Romain Gary, entre deux langues, le français et l'anglais. L'incipit, déjà mentionné, " JE NE ME PLAIS PAS. Oui » (Huston, 1999a : 11) constitue un reflet des fréquentes « ruptures et fragmentations " présentes dans les quatorze chapitres que composent l'essai analysé. Huston - qui, au même 
moment où elle apprenait le français, découvrit le clavecin et le préféra au piano, instrument qu'elle a laissé de côté et associé à l'abandon de l'anglais (Huston, 1999a : 6465) - attribue ces énoncés au soviétique Sviatoslav Richter qui est considéré comme l'un des plus grands pianistes du XXe siècle. Nonobstant, il nous paraît évident que c'est l'écrivaine francophone "qui "parle", en transcrivant ses mots à lui " (Benert, 2016 : 32). On serait tenté également, comme le suggère cette dernière auteure, à croire qu'il puisse s'agir d'une traduction excessivement littérale du russe. Dans cette langue, "la négation peut/doit être renforcée par une affirmative" (Benert, 2016 : 31). L'apparente erreur de traduction dudit incipit (oui au lieu de non) représente plutôt une anacoluthe, provoquant un effet de surprise et de désorientation chez le lecteur, qui ne peut, en tout cas, comprendre d'emblée ce que veut dire l'écrivaine.

Les confidences de Huston à Leïla Sebbar viennent nous éclairer sur le sens des ces énoncés énigmatiques. L'auteure de Nord perdu écrit à propos de son studio à Paris : J'y suis seule la plupart du temps, je m'y sens tout à fait chez moi, et pourtant toujours un peu dépaysée. C'est sûrement pourquoi j'ai voulu vivre dans le Marais, l'un des quartiers les plus bigarrés de Paris, dans lequel mon étrangéité ne peut jamais s'effacer (Huston et Sebbar, $1986: 254$ ).

Nous pouvons considérer l'étrangéité hustonienne comme la désorientation provoquée par le dépaysement. L'écrivaine emploie la métaphore "nord perdu " pour parler de ce dépaysement, qui devient pour elle l'une des principales forces motrices présentes non seulement dans Nord perdu mais aussi dans beaucoup de ses écrits :

Et moi ? Que proclame-je en choisissant pour l'écriture une terre et une langue étrangères - sinon que je suis [...] «ma propre cause et ma propre fin ", capable de me re-mettre au monde à travers l'art, donnant naissance à moi-même, me débarrassant de tous les déterminismes hérités de mes géniteurs? (Huston, $1990: 129)$.

En conclusion, les énoncés antithétiques de l'incipit in media res de Nord perdu laissent transparaittre la démarche de pensée dialectique de Huston, écrivaine migrante, qui après s'être libérée de son passé, finit par se plaire (se sentir à l'aise) grâce à son enrichissement linguistique et culturel.

\subsection{La surconscience linguistique dans Nord perdu}

Nous allons analyser à présent, selon une approche intertextuelle, les arguments de Huston dans ses essais qui nous dévoileront la surconscience linguistique de l'auteure, et ceci progressivement, au fur et à mesure que nous définissons les corrélats associés à cette surconscience linguistique chez l'auteure. Nous avons déterminé d'étudier Nord perdu avec une méthode intégrative, en tenant compte également des circonstances biographiques de l'écrivaine (contexte de migration). 


\subsubsection{L'interlangue (code-switching)}

Si on entend par interlangue (dans le sens de code-switching), la langue en formation, en gestation, et bien que son origine se trouve dans la didactique des langues étrangères, c'est bien évidemment son application littéraire qui nous intéresse ici. L'interlangue fait référence ici au fait d'écrire deux langues à la fois, comme c'est le cas chez Nancy Huston ou chez Samuel Becket, par exemple, où une langue peut se laisser percevoir sous l'autre. Ainsi, chez Huston, tel le piano sous le clavecin, la comparaison anglais-français, le piano étant associé à la langue anglaise et le clavecin, à la langue française (Huston, 1999a : 64-65), «l'anglais ne laisse pas de faire entendre, sa voix "en transparence", sous le français, par l'intermédiaire, notamment, de diverses références culturelles » (Dion, Lüsebrink et Riesz, $2002: 45$ ).

Examinons donc ce point dans Nord perdu où les expressions anglaises (le plus souvent en italiques) sont abondantes : "To be all abroad", "The true North strong and free ", "which country?" (Huston, 1999a : 12, 14 et 34). On y trouve également des mots anglais intégrés dans des phrases françaises : "Golden Oldies" (Huston, 1999a : 26), "self-conciousness " (Huston, 1999a : 38), " world view» (Huston, 1999a : 51), "God» (Huston, 1999a : 63), "clean no-smoking» (Huston, 1999a : 98) (expressions dont elle dévoile toujours, le cas échéant, l'équivalence en français tout au long du texte), des anglicismes comme notamment, "rocking chairs " (Huston, 1999a : 57), " pick up " (Huston, 1999a : 72 et 114), "boom-boom-boom » (Huston, 1999a : 113), "des steaks " (Huston, 1999a : 113), «barbecue " (Huston, 1999a : 113). En outre, nous pouvons même trouver toute une conversation reproduite en anglais dont Nancy Huston ne fournit pas d'équivalence (Huston, 1999a : 60), sans doute pour rendre plus vivant et humoristique son récit. En outre, elle veut nous faire pénétrer dans son univers mental pour nous prouver qu'elle n'est qu'une "fausse bilingue " (Huston, 1999a : 59) car son attention est plus sélective à l'anglais qu'au français. De même avec "Pass the Ketchup!» (Huston, 1999a : 71), on peut en tirer la même conclusion.

Sur le plan socioculturel, la chanson américaine anglophone y est bien présente, lorsque Huston utilise les mots anglais déjà cités, "Golden Oldies" (Huston, 1999a : 26) pour parler des "vieux tubes" ou "Les Blues» (Huston, 1999a : 82). En outre, elle fait référence à l'écrivain canadien anglophone, Matthew Manera, "A Turning of Leaves" (Huston, 1999a : 24), à Thomas Eliot dans sa citation du début du livre, "Home is where you start from" (Huston, 1999a : 7), et à Henry James "The Jolly Corner» (Huston, 1999a : 109). Il y a aussi, bien d'autres références culturelles, par exemple, "Bill Clinton et Philip Roth, Fine Arts Museum et Boston Harbor, vague de chaleur en Floride et télévangélistes " (Huston, 1999a : 21), "Metropolitan Museum (New-York)»(Huston, 1999a : 101), «les montagnes Rocheuses » (Huston, 1999a : 102), "Mont-réal " (Huston, 1999a : 102), " fleuve Saint-Laurent » (Huston, 1999a : 102), «New-Hampshire» (Huston, 1999a : 102), "Amsterdam Avenue à 
Manhattan " (Huston, 1999a : 103), " des mufffins " (Huston, 1999a : 113) etc., et la charmante narration pleine d'humour du "Kellogg's corn flakes " qui la réconforta dans le pays étranger de sa mère adoptive (Huston, 1999a : 75-76).

D'autres termes anglais y apparaissent mais c'est pour citer par exemple l'interférence (mnésique) bilingue entre les mots anglais-français, les faux-amis (Huston, 1999a : 54), les anglicismes (Huston, 1999a : 58), les connotations des mots (Huston, 1999a : 62), notamment des mots "bracken, leaves, fog" (Huston, 1999a : 62). Comme le souligne Pascale Sardin-Damestoy (2007 : 265) :

Les anglicismes et les gallicismes qui émaillent les textes de Huston [...] sont non seulement des symptômes de la contamination d'une langue par l'autre qui souligne l'arbitraire des mots, mais aussi et surtout des traces palimpsestueuses de l'autre langue temporairement abandonnée.

En guise de conclusion, citons l'épitaphe que, selon David Homel (1996), Nancy Huston s'est choisie, "once abroad, always abroad" (apud Dvorak et Kousta, 2004 : 45), qui comme le précise Dvorak et Kousta (2004 : 45) «signifie à la fois "une fois à l'étranger, pour toujours à l'étranger" et "une fois qu'on est à l'étranger, on a pour toujours perdu le Nord" ".

\subsubsection{La bi-langue ou auto-traduction}

Huston considère qu'il "est important ce qui est traduisible» (Huston, 1999a : 90) et selon elle, "un bon livre, à la différence d'un pavé dans la mare, est presque toujours traduisible » (Huston, 1999a : 91). Pour comprendre les arguments que l'écrivaine déploie sur la bi-langue (ou auto-traduction), il convient préalablement de définir ce qu'on entend par bi-langue. Il s'agit du processus de traduction conscient, ou inconscient, d'une langue à l'autre (El Nossery, 2007). À nouveau, ce qui nous intéresse ici, c'est l'application littéraire de ces expériences de traduction.

Précisons que les problèmes de la traduction, du passage d'une langue à l'autre, sont très présents chez les écrivains migrants. Comme le déclare Farkas (2005 : 38), "l'exilé se met à creuser avec obstination l'espace de l'entre-deux-langues, de l'entredeux-cultures", d'où "la traduction est une errance de l'auteur entre deux langues " (Farkas, 2005 : 42). El Nossery (2007 : 391) ajoute qu'il s'agit en fait d'une...

perpétuelle opération de traduction entre les deux langues, de sorte que l'expression obtenue ne relève pas totalement de la langue d'adoption. Il s'agirait plutôt d'une troisième langue [...] entre le français et l'anglais chez Huston, cherchant à créer justement cette fausse note qui rendrait l'étranger familial et le familier étrange.

D’ailleurs, Nancy Huston, Samuel Beckett, Vladimir Nabokov ou Julien Green figurent parmi les rares écrivains célèbres qui se sont auto-traduits, «leurs écrits illustrent des thèmes communs, parmi lesquels le langage, l'exil, le déracinement [...] » 
(Wilhelm, 2006 : 59). Chez ces écrivains, on trouve l'auto-traduction, non seulement en tant qu'activité de décentrement, mais aussi comme écriture re-créative. Il s'agit d'une écriture comme alternative à l'insuffisance de la traduction (Huston, 1999a : 92).

En fait, cette traduction d'une langue à l'autre exprime les conflits intérieurs des auteurs. Comme le remarque El Nossery (2007 : 391), « le procédé d'auto-traduction mis en pratique par Huston lui permettra d'exprimer et surtout de marquer son identité et ses appartenances multiples ", et un peu plus loin, elle ajoute "les frontières sont ainsi rendues floues entre l'identité et l'altérité » (Huston, 2007 : 394).

Si nous comparons Losing North (2002), l'auto-traduction de Nord perdu, avec son texte original, nous pouvons apprécier que cette traduction consiste plutôt en une adaptation littéraire. Les adaptations temporelles sont fréquentes chez Huston. Après une analyse comparative d'un corpus des deux livres cités, Rinne (2008: 5) affirme qu'il existe " un décalage entre les repères temporels anglais et français ".

Si nous analysons, en guise d'exemple, la traduction d'un fragment, présent dans le chapitre Disorientation (Losing North), nous comprendrons mieux la problématique dont parle l'auteure. Huston traduit ses énoncés «Ici, vous taisez ce que vous fûtes. [...] Là, vous taisez ce que vous faites" (Huston, 1999a : 20-21) par "Here you set aside what you used to be. [...] There you set aside what you've become " (Huston, 2002 : 11). Afin de rester fidèle au texte littéraire de départ, elle respecte la structure syntaxique parallèle des deux énoncés antithétiques (répétition des verbes des propositions principales, des adverbes de lieu et des conjonctions de subordination). Cependant, dans la traduction du verbe faire (employé dans la seconde subordonnée complétive conjonctive), intervient l'adaptation littéraire. Ainsi, dans la version anglaise, Huston traduit ce dernier verbe par become qui laisse sous-entendre aux lecteurs de Losing North, non pas «ce qu'elle fait » (version française) sinon « ce qu'elle est devenue ». L'explication de cette adaptation pourrait provenir d'un souci de l'écrivaine de respecter également l'allitération présente dans le texte original ("fûtes/faites »). En effet, l'écrivaine choisit dans les subordonnées de deux phrases analysées : «become/be ». En revanche, elle se penche pour l'absence de correspondance entre les temps verbaux "vous faites" (indicatif présent) "you've become " (present perfect). Par conséquent, Huston indique de manière subtile qu'elle veut passer sous silence ce qu'elle est devenue à cause du déterminisme culturel du pays d'accueil, dans Losing North. Cependant, dans sa version française, Huston introduit une subtile nuance : elle tait ce qu'elle fait avec (auto-)détermination, et laisse transparaître ainsi sa vision différente selon qu'elle écrive en anglais ou en français.

D'ailleurs, nous pensons que l'auto-traduction mène à se questionner sur les équivalences entre les langues et à y revenir constamment (Wilhelm, 2006). El Nossery (2007 : 394) ajoute que « l'auto-traduction devient ainsi la figure de dédoublement par excellence ». Et, pour Klein-Lataud (1996 : 228), «l'auto-traduction ou la création parallèle peuvent alors apparaitre comme une façon de transcender le clivage, de 
réconcilier les deux moitiés de l'être intérieurement déchiré en faisant cohabiter harmonieusement les deux langues ». À l'opposé des Cantiques des Plaines (1993) qui, n'était que la version française d'un roman écrit en anglais où il s'agissait donc d'un retour à la langue maternelle, Nord perdu aussi fut traduit par l'auteure même, mais du français vers l'anglais (Losing North). Nous constatons donc chez Huston l'existence d'un vrai bilinguisme littéraire car elle écrit tantôt en français, tantôt en anglais.

\subsubsection{Bilinguisme littéraire}

Farkas (2005:38) considère que " chaque langue a toujours un côté intraduisible, secret et impénétrable pour le néophyte. Seul le parfait bilingue détient le secret. Voilà comment l'entre-deux-langues acquiert une signification toute particulière pour le bilingue ». Huston (1995: 269) nous avoue être " condamnée désormais au bilinguisme » et Bessy (2008: 99) l'explique de la sorte : "l'écriture dans deux langues permet une sorte de déblocage identitaire grâce à l'émancipation linguistique offerte par la langue autre ». C'est ce bilinguisme littéraire qui explique qu'au niveau de la production textuelle, on y trouve des processus de traduction chez Huston, tout comme chez Samuel Beckett, par exemple, qu'elle citera dans son essai (Huston, 1999a : 46). Examinons alors le concept de bilinguisme (en général) selon l'opinion de Huston.

L'écrivaine distinguera les vrais bilingues - qui sont ceux qui « apprennent dès l'enfance à maîtriser deux langues à la perfection et passent d'une langue à l'autre sans état d'âme particulier" (Huston, 1999a : 53) - des faux, parmi lesquels, elle se trouverait, et dans son cas concret, rappelons-le une fois de plus, avec l'anglais, comme langue maternelle et le français, comme langue adoptive. Elle va même suggérer quelles places, celles-ci occuperaient dans son cerveau bilingue (Huston, 1999a : 61-62).

En outre, Huston nous confie avec humour qu' " au bout de dix années de vie à l'étranger, loin d'être devenue "parfaitement bilingue", [elle] [s]e sen[t] doublement mi-lingue " (apud Klein-Lataud, 1996 : 228). Le bilinguisme est donc toute co-présence de deux langues. Huston l'explique très bien : "j'écris tantôt en français, tantôt en anglais mais, quelle que soit la langue que je choisis pour écrire un texte particulier, j'ai besoin [...] de me forger une langue à moi " (Huston, 2004 : 32). À ce propos, elle ajoute :

Quelle est-elle ? Elle est située maintenant, je crois, quelque part entre l'anglais et le français, c'est-à-dire que - sans y réfléchir, sans le faire exprès -, je cherche à préserver en français, ce que j'aime de l'anglais (son ouverture, son économie, son insolence) et en anglais, ce que j'aime du français (sa précision, sa sensualité, son élégance) (Huston, 2004 : 32).

Comme il a été dit plus haut, Nancy Huston, comme Samuel Beckett ont pratiqué le bilinguisme littéraire, et celui-ci, simultané, car en effet, ces auteurs ont écrit alternativement en l'une et l'autre de leurs deux langues (anglais et français). Rappelons aussi que de nombreux ouvrages ont été auto-traduits par Nancy Huston, tels que 
Cantique des Plaines (1993), avec Plainsong (1993), où "(sa) vraie vie d'écrivain démarre "(Huston, 2004 : 25) précise-t-elle, ainsi que Limbes/Limbo (2000), petit livre bilingue en hommage au grand expatrié et écrivain polyglotte, Samuel Beckett.

La raison de l'auto-traduction, selon Névine El Nossery (2007 : 395), résiderait dans le fait que :

Les deux textes s'éclairent mutuellement, voire se complètent dans une dialectique d'interaction et d'empiétement réciproque. Et qu'entre les deux langues il y aurait une infinité de langues qui se mêlent, reflétant par conséquent les insuffisances des langues, leurs manques et leurs faiblesses.

Dans le même sens, Huston encore ajoute : « je me suis aperçue que le fait de traduire mes livres, dans un sens ou dans l'autre, les améliorait... » (Huston, 2004 : 25).

\subsubsection{Plurilinguisme littéraire}

À ce stade de la réflexion, remarquons chez Huston l'emploi de plusieurs langues de manière simultanée dans un même livre, ce que Grutman a appelé hétérolinguisme, terme qu'il employa pour parler des phénomènes de plurilinguisme dans les textes littéraires : "la présence dans un texte d'idiomes étrangers, sous quelque forme que ce soit» (Grutman, $1997: 37$ ).

En effet dans la version française de Nord perdu qui centre notre attention ici, l'auteure utilise par exemple, outre l'anglais déjà mentionné, l'italien farniente, però et in petto (Huston, 1999a : 31, 55 et 92), l'espagnol perro et basta, aussi, allemand (Huston, 1999a : 56 et 90), l'allemand Hier ist kein warum (Huston, 1999a : 108), le bulgare guvetch (Huston, 1999a : 95), et même l'islandais fjölskydubönd (Huston, 1999a : 68).

Comme le remarque très bien Lise Gauvin en reprenant les propos d'Édouard Glissant :

Aujourd'hui, même quand un écrivain ne connaît aucune autre langue, il tient compte, qu'il le sache ou non, de l'existence de ces langues autour de lui dans son processus d'écriture. On ne peut pas écrire de manière monolingue. On est obligé de tenir compte des imaginaires des langues (apud Dion, Lüsebrink et Riesz, $2002: 23$ ).

Cependant, Huston conçoit encore la compétence plurilingue selon la tradition monolingue. En effet, selon l'écrivaine, ses compétences linguistiques "loin d'être superposées [...], elles sont distinctes, hiérarchisées" (Huston, 1999a : 61). Ce qui ne correspond pas à l'actuelle définition de compétence plurilingue fournie par le Cadre Européen Commun de Référence pour les Langues (Conseil de l'Europe, 2001) comme un ensemble de compétences linguistiques des sujets parlants qui sont soumises à l'intégration et l'interaction. 
Quoi qu'il en soit, chez Huston, se trouve l'adoption du plurilinguisme, " le polylinguisme à l'intérieur duquel ils (en se référant à Khatibi et Huston) se situent, leur a offert un espace de liberté et une ouverture vers l'expérience du divers " (El Nossery, $2007: 391)$.

\subsubsection{Interférences interlinguistiques}

Huston aborde également une autre manifestation de la surconscience linguistique dans Nord perdu, la problématique des erreurs provoquées par les interférences interlinguistiques, qui peuvent se définir comme "l'utilisation d'éléments appartenant à une langue tandis que l'on en parle ou que l'on écrit dans une autre » (Mackey, 1976 : 397), spécialement celui de l'accent que Huston définit comme « un soupçon » (Huston, 1999a : 33). Elle mentionne par exemple que la simple erreur de genre dans l'accord de l'article avec son substantif suffit pour être mis en évidence. Mais, rappelons qu'elle fait surtout référence, dans Nord perdu, à l'interférence phonologique, et plus spécifiquement à "l'articulation (qui) englobe de nombreuses caractéristiques de l'interférence qui entraînent ce qu'on appelle communément un "accent étranger" " (Mackey, 1976 : 408).

Cependant, l'auteure de Nord perdu nous confie paradoxalement « qu'au fond, [elle] y tient (à cet accent)» (Huston et Sebbar, 1986 : 252). Nous expliquons également, avec Dvorak et Koustas (2004 : 26), qu' " une identité monologique ou indivise l'empêcherait de vivre et de créer, tandis que la division, soit donnée, soit élue, lui est littéralement vitale en tant qu'être créatif ». Ainsi, Huston révèle que "la friction entre [elle]-même et la société qui [l]'entoure [...] [l]'est plus que précieuse, indispensable » (Huston et Sebbar, $1986: 252$ ).

Huston nous révèle donc ses difficultés avec le français dans la conversation orale, d'où sa préférence pour l'écriture où l'accent ne s'entend pas (Huston, 1999a : 38-39). D'ailleurs, la langue étrangère ne lui permet pas de bavardages ni de péroraisons, et elle l'empêche de se prendre trop au sérieux. En effet, elle parle avec un accent, par exemple lorsqu'elle se met en colère, elle fait rire sans le prétendre.

Puis, Huston aborde le phénomène de la diversité des usages au sein d'une même langue. Elle se reproche un curieux accent en anglais (lorsqu'elle lit des extraits de ses romans à un public anglophone) dont elle ignore la provenance et qui ne lui plait absolument pas : "Pourquoi diable un accent britannique? (Huston, 1999a : 41). Cela laisse sous-entendre qu'elle préfère tout de même l'accent américain à l'européen ! Ce qui révèle une fois de plus, qu'elle y tient à son étrangéité.

D'autre part, l'emploi de mots français dans son anglais constitue le résultat de transferts interlangues négatifs (Wlosowicz, 2010) car, selon Huston, le français a même ruiné sa maîtrise de l'anglais : "je l'avais délaissé trop longtemps, ma langue mère » (Huston, 1999a : 51). 


\subsubsection{Acquisition des langues étrangères}

Elle argumente également dans son ouvrage la difficulté d'acquérir une langue étrangère (Huston, 1999a : 32). Elle y parle également, de l'inné de la langue maternelle et de l'acquis des langues secondes (qui pour elle, seront d'abord, l'allemand et puis le français, principalement). Elle est persuadée que l'imitation occupe une place primordiale dans l'apprentissage de la langue étrangère, elle emploie par exemple, un français, sans ou avec accent québécois, en fonction du lieu où elle se trouve, elle imite donc (Huston, 1999a : 34-36). Elle considère que « quand on s'initie à une langue, on se comporte d'abord comme un comédien "(Castellani, 2001 : 407). Elle remarque aussi qu'au Québec, par exemple, on a de l'anglais dans le français, mais pas en France (Huston, 1999a : 35).

Elle déplore également les dictionnaires qui nous jettent dans la confusion, notamment dans la recherche du sens de perdre le Nord/ to be all abroad, "l'effrayant magma de l'entre-deux-langues, là où les mots ne veulent pas dire, là où ils refusent de dire, là où ils commencent à dire une chose et finissent par en dire une autre " (Huston, 1999a : 13). Elle nous rappelle ainsi l'importance des approches communicatives en didactique de langues étrangères. En effet, les dictionnaires sont insuffisants pour acquérir une langue étrangère, rappelant ainsi l'insuffisance de la méthodologie de grammaire-traduction : apprendre une langue étrangère n'équivaut pas à la traduire (Huston, 1999a: 57). Finalement, elle remarque l'importance de l'apprentissage précoce des langues étrangères : les enfants ont plus de facilité, ils sont plus réceptifs à la langue étrangère et peuvent réellement arriver à la connaître comme les natifs (Huston, 1999a : 58).

Voyons à présent, la lutte et les difficultés dans l'acquisition de la langue française dont Nancy Huston nous fait part dans Nord perdu, reflets de la surconscience linguistique. Elle déclare d'abord que cette surconscience linguistique de l'étranger pour s'adapter est propice à l'écriture et que l'acquisition d'une langue seconde annule le caractère naturel de la première langue où rien n'est donné d'office, d'où une attention extrême aux tournures, aux mots, aux façons de parler (Huston, 1999a : 43).

En outre, à propos des libertés plus grandes par rapport à la tradition littéraire $\mathrm{du}$ pays d'accueil que les écrivains francophones adoptent, Huston considère que " cette faculté naîtrait d'une capacité supérieure d'attention à la langue acquise " (apud Dion, Lüsebrink et Riesz, 2002 : 60). Elle se reproche que son français soit toujours de l'appris. Elle avoue, par exemple, son écoute pathologique de la langue, coïncidence sonore ou, du côté du style, sa résistance à manier le passé simple "trop guindé et prétentieux " (Huston, 1999a : 45).

Elle évoque également dans Nord perdu les difficultés lexicales en français (interférences interlinguistiques, déjà mentionnées plus haut), les trous de mémoire, les faux amis, les mots à usage sporadique qui nomment un objet précis et qu'elle ne retient pas, les oublis du sens des mots, surtout des noms. Selon le mari de l'écrivaine (Tzvetan 
Todorov), " ce sont eux que, dans la langue étrangère, l'on perd en premier - de même que, dans la langue maternelle, tout un chacun voit se dérober avec l'âge les noms propres » (Huston, 1999a : 56). De plus, l'emploi du passé simple notamment lui pose un problème au moment de maintenir une conversation (Huston, 1999a : 58). De même, elle éprouve des difficultés avec l'emploi d'anglicismes, de certaines expressions argotiques, d'abréviations ou encore de liaisons (Huston, 1999a : 58-59). Huston présente des capacités métalinguistiques disproportionnelles en anglais et en français qui se traduisent par une plus grande attention sélective à la langue anglaise face à la française. Elle cite l'exemple du récit en langue française du mariage de son amie dans un bar où elle n'a pu écouter que la grossière conversation en anglais du reste des clients (Huston, 1999a : 60). Elle déclare cependant la préférence du français dans certaines activités, par exemple, dans la conversation intellectuelle et de l'anglais dans d'autres, telles que pour " $[\mathrm{s}] \mathrm{e}$ défouler, jurer, chanter, gueuler, [s]e laisser aller au pur plaisir de la parole" (Huston, 1999a : 61). Elle parle du problème fort intéressant de l'absence subjective de connotation dans les mots exprimés en langue étrangère (Huston, 1999a: 62-64). Cependant, cette neutralité est idéale pour Huston dans son écriture. En effet, elle écrit :

Le je que j'employais dans mes essais, totalement nu et intime, sans protection aucune, était par ailleurs l'un des effets du savoir déraciné : en effet, cette impudeur était facilitée par l'emploi de la langue étrangère, en partie parce qu'elle n'était pas comprise par mes parents (fantasmatiquement, du moins), mais surtout parce qu'elle n'était justement pas, pour moi, de l'ordre de l'intime. Je pouvais écrire avec tranquillité, voire avec indifférence, des choses qu'il m'eût été impossible de révéler dans ma langue maternelle (Huston, $2004: 22-23$ ).

\subsection{L'étrangéité chez Huston}

Afin d'introduire la seconde partie de notre travail, rappelons que Huston s'est établie en France depuis les années 1970. C'est à cette époque qu'elle éprouve un sentiment de dépaysement. Nancy Huston préfère employer le terme d'étrangéité - un néologisme qu'elle a elle-même contribué à forger d'après Jane Elisabeth Wilhelm (2006) - . Rappelons que ce fut dans ses correspondances sur le thème de l'exil qu'elle a maintenues avec Leïla Sebbar qu'elle avoua y tenir à cette étrangéité (Huston et Sebbar, $1986: 254)$.

Deleuze et Guatarri (1975) ont créé le terme de territorialisation, comme le rapport d'appartenance identitaire de l'individu ou de la collectivité tel qu'il se manifeste à travers le langage et de reterritorialisation pour faire référence au processus compensatoire par lequel le sentiment identitaire retrouve un certain sens. Comme nous le verrons tout de suite, dans Nord perdu, l'écrivaine fait référence aux expatriés (déterritorialisés) et aux impatriés (territorialisés). 
Dans cet essai autobiographique, Huston défend l'importance du lieu où l'on passe les «vingt-cinq premières années" (Huston, 1999a : 16) de la vie pour marquer l'appartenance à la collectivité humaine où l'on vit, et ceci marque de façon indélébile. "L'enfance est toujours en nous", ajoute-t-elle (Huston, 1999a : 17). Huston précise que « [...] personne ne peut se prétendre sans enfance [...] période de vulnérabilité et d'impressionnabilité absolues" (Huston, 1995 : 218). Elle introduit la distinction déjà mentionnée entre les impatriés et les expatriés, et entre les monolingues et les bilingues.

En outre, elle nous transmet ses réflexions sur la relativité culturelle, l'intercommunication, l'interculturalité. Elle est convaincue que ses enfants pourront se sentir plus français qu'elle (Huston, 1999a : 16), et remarque également que les difficultés pour se sentir français seront plus grandes pour les enfants des Noirs, par exemple, comparés aux Canadiens, notamment. Huston est donc persuadée que ses enfants seront plus aptes à adopter les valeurs de la société d'accueil et évoque « la difficulté de se faire entendre pour la première génération d'immigrants et du difficile dialogue à établir entre cette génération et la suivante plus aptes à adopter les valeurs de la société d'accueil "(Dion, Lüsebrink et Riesz, 2002 : 31). Comme nous l'avons mentionné plus haut, Huston considère que l'enfance est toujours en nous. Elle nous décrit, à ce sujet, une discussion qu'elle a eue avec un de ses lecteurs qui ne l'entendait pas ainsi. L'écrivaine plaint cette personne : "pauvre qui n'était qu'adulte. (Pauvre, je veux dire volontairement appauvri). Pas en exil celui-là » (Huston, 1999a : 18). Voici un extrait de cette conversation :

- Mais je ne suis pas un enfant, moi ! protesta-t-il.

- Bien sûr que si, vous l'êtes ; on est tous nos âges à la fois, vous ne croyez pas? L'enfance, c'est comme le noyau du fruit : le fruit en grandissant, ne devient pas creux! Ce n'est pas parce que la chair s'épaissit autour de lui que le noyau disparaît...

- Je suppose, lança alors mon interlocuteur excédé, qu'il est interdit de ne pas être d'accord (Huston, 1999a : 18).

D’ailleurs, elle déplore que les expatriés soient toujours exposés aux questions stupides, comme on peut l'apprécier lors de sa réponse à la question "vous sentez-vous française maintenant? " (Huston, 1999a : 16). "Jamais français[e] parce que personne ne peut [me] donner une enfance française ", répondra Huston (1999a : 17). En outre, Huston conçoit les écrivains migrants comme s'ils étaient elle-même, car ils " ne sont ni enracinés ni déracinés; souvent du reste, ils perçoivent l'idée même de racines comme une illusion, voire une métaphore dangereuse. Ils ne sont ni sédentaires, ni nomades. Ils sont exilés " (Huston, 2004 : 59), reterritorialisés, pourrait-on ajouter. Elle nous donne un peu plus loin la clé d'interprétation pour notre sujet: "l'écrivain en exil, même volontaire, loin d'être "chez lui partout où il pose le pied", n'est chez lui nulle part" (Huston, $2004: 61$ ). 
Ainsi, parmi les problèmes que rencontrent les expatriés, Huston remarque les différences entre génération dues à l'exil (Huston, 1999a: 22-23), et l'écrivaine mentionne également le "choc culturel » que rencontre les migrants. Elle parle de la coupure qu'a constitué son exil social qu'elle argumente avec un extrait de Matthew Manera A Turning of Leaves (Huston, 1999a : 23-24). Elle déclare que quitter son pays, c'est conduire le reste de son existence dans une culture et une langue, jusque-là étrangères, et s'installer à tout jamais dans l'imitation, le faire-semblant, le théâtre (Huston, 1999a : 30). D'autre part, l'expatrié reste inféodé à la famille sur le plan imaginaire (Huston, 1999a: 28). Rappelons que, selon l'écrivaine, dans le pays d'adoption " vous taisez ce que vous fûtes " (Huston, 1999a : 20), en revanche, dans le pays trahi "vous taisez ce que vous faites" (Huston, 1999a : 21). En effet, lors de la confrontation dans le pays d'accueil avec la langue et culture étrangères, les traits culturels de l'expatrié sont découverts et mis en évidence (Huston, 1999a: 31). Comme nous y avons déjà fait allusion, Huston nous révèle la difficulté d'acquisition d'une langue étrangère (Huston, 1999a : 32), elle parle de l'inné de la langue maternelle et de l'acquis des langues secondes, et des problèmes, des erreurs et des interférences des étrangers qui trahissent leur étrangéité (Huston, 1999a : 33-34). En nous parlant du divorce de ses parents et de son exil en Allemagne chez sa mère adoptive, elle nous raconte d'une manière très drôle l'anecdote très significative, "des corn flakes " où, étant en Allemagne et devant le dépaysement de tout ce qui l'entourait, les banals « corn flakes » américains ont suffi pour la sortir de sa désolation (Huston, 1999a : 75-76).

Elle argumente également l'isolement des étrangers, la barrière que constitue la langue : "il suffit qu'on prononce un seul mot et (les autochtones) le savent : on n'est pas d'ici " (Huston, 1999a : 77). Réduit "à l'infans» (Huston, 1999a : 78), privé de parole, réduit au silence, on paraît "dumb» (Huston, 1999a : 78), "chaque menu détail [de la vie quotidienne] est une montagne " (Huston, 1999a : 78). Elle cite, dans l'œuvre analysée, l'anecdote lors d'une demande d'itinéraire à un passant : si on ne comprend pas quelqu'un, on a une vision négative de cette personne, et c'est le contraire, si on sait se communiquer avec elle ; de là que l'étranger soit, ou plutôt paraisse, "bête" (Huston, 1999a : 79) et "barbare" (Huston, 1999a : 79). Pour illustrer ceci, elle nous raconte dans son essai son "rêve éveillé " (Huston, 1999a : 8081) à propos de la détresse de Lepen dans un pays étranger où personne ne le comprenait et où il se sentait inférieur, dans une situation désagréable à cause de la langue qu'il ne connaissait pas, il ne comprenait pas la langue de ses habitants et s'en trouvait dès lors exclu.

Elle nous rappelle également, l'existence de racisme explicite en France (Huston, 1999a : 82) et nous dévoile un racisme implicite au Canada (Huston, 1999a : 83-84), comme le lui déclare son frère qui vit en Amérique du Nord.

Elle nous avoue aussi, son ignorance de la culture multiculturelle canadienne (Huston, 1999a : 85). Ainsi, elle argumente que ce qui est proche a de l'importance 
mais pour l'étranger, ceci ne va pas de soi car ses proches sont loin (Huston, 1999a : 86-88). Dans les pages suivantes de cet essai, elle rappelle les problèmes de l'expatrié. Au début, peut-être se sent-il « touriste [...] [et goûte ainsi de] la magie du lieu étranger et prestigieux " (Chaulet Achour, $2006: 44$ ). Cependant, cela se transforme très vite en souffrance, en spleen. Ainsi, survient l'espacement des communications avec les siens, et de nouveaux amis remplacent les anciens avec l'oubli des amis d'antan, une nouvelle famille formée. Les étrangers deviennent alors des « compatriotes » (Huston, 1999a : 88), une nouvelle culture trouvée.

De plus, lors des retours de l'écrivaine au Canada anglophone, celle-ci se sent étouffée à la fois par sa langue maternelle et par sa mère patrie. Elle arrivera à conclure que le patriotisme est un attachement arbitraire. Croire que la mémoire est inviolable n'est pas exact car l'insuffisance de la mémoire est argumentée avec l'exemple de la disparité entre ses souvenirs des mêmes événements passés et ceux d'une amie d'adolescence, d'où l'importance d'alimenter les souvenirs (Huston, 1999a : 96-99). Les expatriés se rendent compte que nous sommes des "livre[s] aux pages arrachées " (Huston, 1999a : 100). Ainsi, l'écrivaine doublement déracinée, par le pays et par la langue, expose l'un des principaux problèmes de l'exilé, la culpabilité qu'il éprouve d'avoir quitté son pays (Huston, 1999a : 15, 89) et celle d'avoir choisi une autre langue. L'exil devient synonyme de rupture (Huston, 1999a : 20-22). Névine El Nossery (2007 : 389) remarque " que la traversée transculturelle soit géographique, linguistique ou symbolique, volontaire ou forcée, elle s'accompagne toujours de rupture, de nostalgie et de carence, donc de perte que seule l'écriture peut combler ".

Par conséquent, surviennent les questions sur l'identité et le débat identitaire de l'exilé : "qui sommes-nous, alors?", "qui est-on alors?", " qui suis-je? " (Huston, 1999a : 52, 89 et 108). Huston se demande qui elle est, si elle n'a pas " les mêmes pensées, fantasmes, attitudes existentielles, voire opinions dans une langue et dans une autre» (Huston, 1999a : 52). Farkas (2007: 35) précise à ce sujet :

Au-delà de l'expression linguistique, la perte de l'Est et du Grand Nord semble représenter le même drame pour un écrivain puisqu'il en sort non seulement mutilé mais aussi coupable. $\mathrm{Mu}$ tilé à cause d'une enfance et d'une langue perdues, et coupable puisque le sentiment de culpabilité d'avoir quitté une collectivité ne lâchera jamais celui qui se met à écrire dans une autre langue et dans un autre endroit. Par conséquent, les romans d'exilés ont un caractère autobiographique accentué.

"Je l'ai trahi, et je l'ai perdu» (Huston, 1999a : 15), se lamentera Huston ou " traîtresse du Grand Nord»(Huston, 1999a : 84), se jugera-t-elle.

Quoique dans la vie de toute personne, il y ait des changements, il lui reste une identité stable, mais pas pour l'expatrié; d'où le fantasme des alter ego (Huston, 1999a : 109-115). Todorov (1998: 22), écrivain migrant également, ajoute "de ahí que 
persistiera en mí la impresión de que una de mis vidas debía de ser un sueño ", ou encore : "su alter ego, la variante de sí mismo que había permanecido en el lugar..." (Todorov, 1998 : 23). En outre, Huston fait également allusion à Henry James avec la pseudo-conviction de l'existence d'un alter ego dans le pays quitté (Huston, 1999a: 109). L'écrivaine donne aussi raison à Milan Kundera (auteur bilingue) qui pense qu'il est inconcevable de n'avoir qu'une seule vie (Huston, 1999a: 115). En réalité, l'existence d'un alter ego peut se justifier pour pallier les remords et la culpabilité d'avoir abandonné et laissé une culture. "Comment faire comprendre à des Européens ce que signifie l'absence totale de ce qu'ils chérissent par-dessus tout: l'enracinement ?" (Huston, 1995 : 200), se plaindra l'écrivaine née à Calgary, qui se déclare " apatride et antipatriotique " (Huston, $1995: 201$ ).

Comme c'était le cas avec la surconscience linguistique, l'originalité et l'optimisme de ces écrivains sera celui de surmonter le côté négatif de l'exil. Voyons ceci chez l'écrivaine. "Je ne plais pas » dit Sviatoslav Richter (apud Huston, 1999a : 11), et laisse sous-entendre également Nancy Huston, quand elle fait allusion à Romain Gary. Pourquoi ? L'explication est simple : parce qu'ils sont étrangers. Cependant, de là leur vient paradoxalement leur grandeur. Huston parle "de la supériorité de l'exilé, s'expliquant par le fait qu'il dispose d'une expérience socio-politique différente de celle des natifs" (Farkas, 2005: 44). Les expatriés apprennent beaucoup à propos de la condition humaine alors que les autres ne s'en rendent pas compte. Les expatriés sont "riches de leurs identités accumulées et contradictoires» (Huston, 1999a : 18).

Nous avons vu plus haut le problème de l'interférence provoquée par l'accent. En revanche, Nancy Huston considère aussi et surtout, son côté positif ; elle a un préjugé positif envers l'accent. De plus, celui qui connaît deux langues et deux cultures a, selon Huston (1999a : 37), "toutes les chances d'être quelqu'un de plus "civilisé", de moins péremptoire que les monolingues impatriés ». En ce qui concerne Huston, écrire en langue étrangère lui permet une plus grande liberté dans son français. Elle passe, par exemple, du registre soutenu au familier abruptement (comme le lui reprochent certains lycéens), elle a ainsi plus de liberté à transgresser les normes et attentes de la langue française (Huston, 1999a : 47). Cette faculté naîtrait selon elle, d'une capacité supérieure d'attention à la langue acquise. Elle nous révèle également " son allergie à l'égard des "syntagmes figés", [s]on goût prononcé pour les parenthèses, les deux points, les points-virgules et les phrases un peu trop longues " (Huston, 1999a : 48). D'ailleurs, Huston (1999a : 50) " os[e] la fiction" et écrit enfin son premier roman en 1981. Elle se détache alors de son maître qui concevait la Littérature " en termes de structure[s] (linguistiques), et non plus en termes d'art " (Barthes, 1953 : 45), et qui considérait le fait que le narrateur ait à se cacher derrière le personnage narrateur trop fictif et difficile à assumer. De plus, elle retourne à la langue anglaise assoiffée d'innocence théorique (Huston, 1999a : 50-51), afin que rien "n'aille complètement de soi »(Huston, 1995 : 202). 
L'écrivaine parle également de l'illusion du mythe de l'auto-engendrement et de la présence en soi du passé et de la lignée. En principe, Huston ne partage pas cette idée, car c'est avec sa langue et sa culture étrangères (francophones) qu'elle est parvenue à son autodétermination (Huston, 1999a : 68-69). Fidèle à son féminisme, Huston aventure avec des arguments assez convaincants que les femmes ont une conception plus souple de leur identité (Huston, 1999a : 93), elle considère qu' " "être français" est une identité parmi d'autres"(Huston, 1999a : 95), et que les exilés ont l'avantage de le savoir. L'écrivaine précise : "en revêtant mon masque francophone, en m'installant dans une culture étrangère, qu'ai-je fait d'autre que de me choisir libre et autonome " (Huston, 1999a : 68). "Pourquoi l'arbitraire lieu de ma naissance aurait-il des droits sur mes désirs actuels?" (Huston, 1995 : 201), se demandera-t-elle. Malgré tout, sur le plan physique (Huston, 1999a : 69-70), l'influence de l'inné reprend force passé la quarantaine, et au niveau caractériel, revient ce que nous repoussions préalablement (Huston, 1999a : 71-72). De là que, l'auto-engendrement ne soit qu'une illusion, un mythe (Huston, 1999a : 72-73) et que nous finissions par ressembler "corps et âme " (Huston, 1999a : 72) à nos géniteurs et au peuple d'où nous sommes issus. Huston conclut que "nous ne sommes entièrement libres que dans nos désirs, et non dans nos réalités "(Huston, 1999a: 73).

En outre, on pourrait parler de multiples cultures (temps-espace) chez Huston (Huston, 1999a : 102-104) et de "multiples identités embrassées " (Huston, 1999a : 105), " puisque je peux être concernée par les problèmes de deux pays, je le suis par ceux de tous les pays " (Huston, 2004 : 61). Encore une fois, l'allusion à Romain Gary apparaît (Huston, 1999a : 105). De plus, elle sent qu'elle partage avec son frère, à qui elle dédicace Nord perdu (rappelons-le), une culture commune (Huston, 1999a : 104).

Dans l'avant-dernier chapitre de Nord perdu, intitulé "Les autres soi I" (Huston, 1999a : 102), Huston fait l'éloge de la littérature (spécialement, du roman), "genre humain par excellence" (Huston, 1999a : 107), dira-t-elle, qui permet de dépasser les limites du moi ou plutôt des moi (Huston, 1999a : 106). Et le roman, notamment, lui donne la liberté "d'aller ailleurs et d'être autrui dans [sa] tête " (Huston, 1999a : 105) ce qui permettra aux romanciers (et aux lecteurs, selon Huston) de ne pas se contenter d'une identité conférée à la naissance.

Rappelons que la question de l'identité est récurrente chez Huston (Huston, 1999a : 52, 89 et 108). "Qui sont-ils ? Des “étrangers à eux-mêmes” (Kristeva, 1988), des êtres "dépaysés" (Todorov, 1998) ? " (Dion, Lüsebrink et Riesz, 2002: 61) reprend Anne-Rosine Delbart (2005). Ou encore, comme le fait Ken Bugul, écrivaine sénégalaise francophone, elle considère qu'elle est " una extrańa en su propio pueblo entre sus familiares y amigos, tal como lo ha sido en Francia " (apud Mallol de Albarracín, 2017 : 66). Cependant, Nancy Huston s'empresse d'écrire «l'histoire de ma vie adulte est celle d'une quête, non pas d'identité, mais d'intensité" (Huston, 1995 : 200). Elle ajoutera «voici dix ans, j'ai publié dans les Temps modernes un texte 
intitulé "la rassurante étrangeté", dans lequel j'exposais les joies de cette virginité culturelle, les avantages non pas du déracinement, mais de l'a-racinement " (Huston, 1995 : 217). Une fois de plus, elle se réfère aux avantages de la reterritorialisation. Et, avec la théorie des "world views" (Huston, 1999a: 51), Huston nous rappelle l'hypothèse de Whorf-Sapir, présentée par Harry Hoijer en 1954 (apud Fromkin, 1977 : 173), selon laquelle c'est la langue qui impose à la communauté sa vision du monde : « je n'étais plus moi-même non plus. Non seulement on prononçait mon nom d'une autre façon, mais j'étais brusquement plus aimable, même à mes propres yeux » (Huston, 1995 : 265).

\section{Conclusions}

Nancy Huston a donc adopté une autre langue que sa langue maternelle comme langue littéraire. Ce choix d'écrire dans une langue autre a entraîné une surconscience linguistique qui provient "d'un sentiment d'étrangeté dans la langue, redoublée lorsqu'il s'agit de ceux parmi les écrivains que l'on désigne sous le nom d'écrivains migrants" (Dion, Lüsebrink et Riesz, 2002 : 25), et qui la condamne, en tant qu'écrivaine, à penser la langue et à la situer entre "l'intégration pure et simple aux codes de la langue française et la valorisation excessive de l'exotisme, comme il est partagé entre le tourment du langage et l'imaginaire des langues » (Dion, Lüsebrink et Riesz, $2002: 25$ ).

Ainsi, dans la première partie de ce travail, ce fut cette « conscience aiguë de la langue comme objet de réflexion, d'interrogation, d'enquête mais aussi de transformation et de création " (Gauvin, $2000: 209$ ) qui a été contrastée et mise en évidence chez Huston dans Nord perdu, essentiellement. Et dans la seconde partie, nous avons vérifié chez Huston l'étrangéité qui l'a rendue libre de choisir sa langue d'écriture (Huston, 1990).

En guise de conclusion, nous pouvons affirmer, à l'instar de El Nossery (2007 : 390), qu'avec Nord perdu, nous nous trouvons devant " une écriture susceptible d'exprimer la pensée biculturelle de l'auteur, traduisant une quête identitaire transscripturale qui passe nécessairement par la langue ou les langues, voire les cultures qui les traversent». Et de se demander à propos de Huston : "l'insécurité linguistique transformée en motivation d'écriture, n'est-ce pas là un exemple éloquent de cette surconscience qui habite tout écrivain dont la langue est déterritorialisée ?" (Dion, Lüsebrink et Riesz, 2002 : 30).

Nancy Huston appartient donc à ces écrivains que l'on peut appeler francophones car "leur pays c'est l'écriture " (Huston, $2004:$ 65), "ce sentiment de non-appartenance induit par le changement de langue serait pour les auteurs bilingues à la fois une source de créativité littéraire originale mais aussi une source de renouveau identitaire thérapeutique" (Bessy, 2008: 92). En définitive, on pourrait dire que Nancy Huston s'identifie avec les " exilés du langage " (Delbart, 2005), comme son 
frère (parlant français aux États-Unis) ou bien d'autres émigrés, comme par exemple, son mari Tzvetan Todorov (célèbre linguiste Bulgare, ayant vécu à Paris), formant un couple qui s'est "transculturellement" (Todorov, 1998 : 27) enrichi. Et elle paraît considérer, comme l'écrivain bulgare: "lo que hay que temer y deplorar es la desculturación en sí misma, degradación de la cultura de origen, aunque pueda ser compensada por la aculturación, adquisición progresiva de una nueva cultura, de que todos los seres humanos son capaces" (Todorov, 1998 : 26). Les propos suivants, qui font allusion à la reterritorialisation ou transculture, s'appliquent également à l'exilée de l'Ouest :

El hombre desarraigado, arrancado de su marco, de su medio, de su país, sufre al principio, pues es más agradable vivir entre los suyos. Sin embargo, puede sacar provecho de su experiencia. Aprende a dejar de confundir lo real con lo ideal, la cultura con la naturaleza (Todorov, $1998: 29$ ).

Nous pouvons conclure que la surconscience linguistique - « je remercie la langue française de tout ce qu'elle a fait pour moi ", révélera Huston (1995 : 219) - et l'étrangéité ont été vitales pour l'écrivaine. Comme elle le révèle elle-même, " pourtant, je suis étrangère et je tiens à le demeurer, à toujours maintenir cette distance entre moi et le monde qui m'entoure, pour que rien de celui-ci n'aille complètement de soi : ni sa langue, ni ses valeurs, ni son histoire" (Huston, 1995 : 202), convaincue que "l'identité est toujours un leurre " (Huston, 1999a : 44). Selon Nancy Huston : " c'est pour cela que je suis devenue écrivain, parce qu'il y avait dans ma vie quelque chose d'incompréhensible qui requérait un immense et perpétuel effort d'imagination pour tenter de le comprendre» (apud Argand, 2001 :35). Et sans doute, grâce à sa surconscience linguistique et à son étrangéité, est-elle arrivée à être ce qu'elle est aujourd'hui, une prolifique écrivaine francophone de grande renommée qui fait partie de ces écrivains trans-européens (Oktapoda, 2017 : 127-128). Il est incontestable que "les créations en français des auteurs venant de l'extérieur de la France sont des contributions très enrichissantes à la littérature française » (Zbanț, 2019 : 150).

\section{RÉFÉRENCES BIBLIOGRAPHIQUES}

ALBERT, Christiane [dir.] (1999) : Francophonie et identités culturelles. Paris, Karthala.

ARMEL, Aliette (2018): "Huston Nancy", in Dictionnaire des Écrivains Francophones. Boulogne-Billancourt, Encyclopaedia Universalis, 165-166.

ARGAND, Catherine (2001) : "Entretien : Nancy Huston». Lire, 293, 31-35. URL : https:/www.lexpress.fr/culture/livre/nancy-huston_804287.html

BARTHES, Roland (1953) : Le degré zéro de l'écriture. Paris, Seuil. 
BENERT, Britta (2016) : «Plurilinguisme et migrations dans Nord Perdu de Nancy Huston ». Carnets : Revue électronique d'études françaises, $7: 2$, 28-41. URL: https://ler.letras.up.pt/uploads/ficheiros/14199.pdf

BESSY, Marianne (2008) : Vassilis Alexakis : Exorciser l'exil. Déplacements autofictionnels, linguistiques et spatiaux. Thèse doctorale. URL: https://digitalcommons.lsu.edu/gradschool_dissertations/3456/

CASTELLANI, Jean-Pierre (2001) : "La langue de l'autre ». Littérature et Nation, 24, 405-412.

Chaulet ACHOUR, Christiane (2006) : "Nancy Huston et ses langues ». Le Français dans le monde. Recherches et applications, 39, 42-51.

CONSEIL DE L'EUROPE (2001) : Un Cadre européen commun de référence pour les langues: apprendre, enseigner, évaluer. Strasbourg, Division des politiques linguistiques du Conseil de l'Europe. URL : https://rm.coe.int/16802fc3a8

DAY, Loraine (2007) : "Trauma and the bilingual subject in Nancy Huston's L'empreinte de l'ange ». Dalhousie French Studies, 81, 95-108.

DELBART, Anne-Rosine (2005) : Les exilés du langage : Un siècle d'écrivains français venus d'ailleurs (1919-2000). Limoges, Presses Universitaires de Limoges.

Deleuze, Gilles \& Félix, GuATTARi (1975) : Kafka. Pour une littérature mineure. Paris, Les Éditions de Minuit.

DION, Robert ; Hans-Jurgen LÜSEBRINK \& János RIESZ (2002) : Écrire en langue étrangère. Interférences de langues et de cultures dans le monde francophone. Québec, Éditions Nota Bene.

DVOraK, Marta \& Jane Koustas [dir.] (2004) : Vision/Division. L'ouvre de Nancy Huston. Presses de l'Université d'Ottawa. URL: https://ibrary.oapen.org/bitstream/id/3ebe8ca5-216e-4b55-a549-58276f89febb/579208.pdf

EL NOSSERY, Névine (2007) : «L'étrangeté rassurante de la bi-langue chez Abdelkébir Khatibi et Nancy Huston ». Contemporary French and Francophone Studies, 11 : 3, 389-397.

ELIOT, Thomas Stearns (1940) : East Coker (Four Quartets). Londres, Faber and Faber.

FARKAS, Jenö (2005) : "L'écrivain désorienté ou les aspects de l'estitude (Dumitru Tsepeneag, Nancy Huston, Katalin Molnár)». Nouvelles Études Francophones, 20 : 1, 35-46.

FROMKIN, Victoria (1977) : "Harry Hoijer». Language, 53: 1, 169-173. URL: https://www.jstor.org/stable/413060

FOLGUERÁ, Juan José (1993) : "Los four quartets de T. S. Eliot: "situación” y "traducción” ". Cauce, 16, 243-276.

GAUVIN, Lise (1997) : L'écrivain francophone à la croisée des langues. Entretiens. Paris, Karthala.

GAUVIN, Lise (2000) : Langagement. L'écrivain et la langue au Québec. Montréal, Boréal.

GAUVIN, Lise (2003) : «Autour du concept de littérature mineure. Variations sur un thème majeur ", in Jean-Pierre Bertrand et Lise Gauvin [dir.], Littératures mineures en langue majeure. Montréal, Presses de l'Université de Montréal, Peter Lang, 19-40. URL: https://books.openedition.org/pum/15718 
GRUTMAN, Rainier (1997) : Des langues qui résonnent. L'hétérolinguisme au XIX' siècle québécois. Québec, Fides - CÉTUQ.

Homel, David (1996) : "Once Abroad, Always Abroad - David Homel speaks with Nancy Huston». Books in Canada: The Canadian Review of Books, $25: 3$.

Huston, Nancy (1990) : Journal de la création. Paris, Seuil.

HUSTON, Nancy (1993a) : Plainsong. Toronto, HarperCollins.

Huston, Nancy (1993b) : Cantique des plaines. Arles, Actes Sud.

Huston, Nancy (1995) : Désirs et réalités : textes choisis 1978-1994. Montréal/Paris, Leméac.

Huston, Nancy (1999a) : Nord perdu (suivi de Douze France). Arles, Actes Sud.

Huston, Nancy (1999b) : Prodige : polyphonie. Arles, Actes Sud.

Huston, Nancy (2000) : Limbes/Limbo. Montréal/ Arles, Leméac/Actes Sud.

HUSTON, Nancy (2002) : Losing North : Musings on Land, Tongue and Self. Toronto, McArthur \& Company

Huston, Nancy (2004) : Ames et corps : textes choisis 1981-2003. Montréal / Arles, Leméac / Actes Sud.

HUSTON, Nancy \& Leïla SebBAR (1986) : «Lettres parisiennes ». Communications, 43, 249265. DOI : https://www.persee.fr/doc/comm_0588-8018_1986_num_43_1_1 650

KLeIN-LATAUd, Christine (1996) : «Les voix parallèles de Nancy Huston ». Traduction, Terminologie, Rédaction, 9: 1, 211-231. URL : https://www.erudit.org/en/journals/ttr/1996-v9-n1-ttr1484/037245ar.pdf

LIÉBOIS, Katrien (2018) : "Surconscience linguistique et ironie. Poétiques de la résistance de l'écrivain francophone au Maghreb et en Afrique subsaharienne ", in Christine Meyer \& Paula Prescod [ed.], Langues choisies, langues sauvées. Poétiques de la résistance. Wurtzbourg, Königshausen \& Neuman, 327-341.

MACKEY, William Francis (1976) : Bilinguisme et contact des langues. Paris, Klincksieck.

MALLOL DE AlBARRACÍN, Claire (2017) : « Ken Bugul: extranjera también en su propia casa », in Ana María Gentile ; Claudia Moronell ; María Julia Zaparart ; María Leonor Sara \& María Paula Salerno [comp.], Miradas sobre la literatura en lengua francesa: Hospitalidad, extranjería, revolución y diálogos culturales. XXX Jornadas de Literatura Francesa y Francófona. La Plata, Universidad Nacional de la Plata, 65-73.

OKTAPODA, Efstratia (2017) : «Frontierland ou comment traverser les frontières : Vassilis Alexakis, identité européenne et altérité nationale». Carnets: Revue électronique d'études françaises, 11, 127-136. DOI : https://doi.org/10.4000/carnets.2356

RINNE, Noëlle (2008) : «La tierce-langue de Nancy Huston ». Crisolenguas, 1 : 1, 1-9. URL: https://crisolenguas.uprrp.edu/Articles/La\%20tierce\%20langue $\% 20 \mathrm{de} \% 20 \mathrm{Nancy-}$ \%20Huston.pdf

RuSSELl, Bruce (1990) : «True North». The Massachusetts Review, 31 : 1/2, 81-85.

SARDIN-DAMESTOY, Pascale (2007) : «Samuel Beckett / Nancy Huston ou le bilinguisme de malentendus en contrefaçons : deux expériences similaires ? ", in Axel Gasquet et 
Modesta Suárez [dir.], Écrivains multilingues et écritures métisses: l'hospitalité des langues. Clermont-Ferrant, Presses Universitaires Blaise Pascal, 267-270.

TODOROV, Tzvetan (1998) : El hombre desplazado. Madrid, Taurus-Santillana Ediciones.

WILHELM, Jane Elisabeth (2006) : «Autour de Limbes/Limbo : un hommage à Samuel Becket de Nancy Huston ». Palimpsestes, 18, 59-85. DOI : https://doi.org/10.4000/palimp$\underline{\text { sestes. } 547}$

WLOSOWICZ, Teresa María (2010) : «Le transfert et les interférences entre L1, L2 et L3 dans la production des cognates aux terminaisons différentes ». Synergies Espagne, 3, 159170.

ZBANT, Ludmila (2019) : « Le dialogue des langues-cultures dans la littérature francophone et les contextes de sa traduction ». Studia Universitatis Moldaviae, 124 : 4, 146-150. 